\title{
A Prototype of Google Interfaces Modified for Simplifying Interaction for Blind Users
}

\author{
Patrizia Andronico, Marina Buzzi \\ IIT, National Research Council \\ via Moruzzi, 1, 56010 Pisa, Italy \\ +39-050-3152090, +39-050-3152631 \\ Patrizia.Andronico@ iit.cnr.it \\ Marina.Buzzi@iit.cnr.it
}

\author{
Carlos Castillo \\ Università di Roma "La Sapienza" \\ via Salaria 113, 00198 Roma, Italy \\ +39-064-9918344 \\ Castillo@dis.uniroma1.it
}

\author{
Barbara Leporini \\ ISTI, National Research Council \\ via Moruzzi, 1 -56010 Pisa, Italy \\ $+39-050-3152034$ \\ Barbara.Leporini@isti.cnr.it
}

\begin{abstract}
In this study we present a SW prototype developed within the framework of a research project aiming at improving the usability of search engines for blind users who interact via screen reader and voice synthesizer. Following the eight specific guidelines we proposed for simplifying interaction with search engines using assistive technology, we redesigned Google user interfaces (i.e. simple search and result pages) by using XSL Transformations, Google APIs and PERL technologies. A remote test with 12 totally blind users was carried out in order to evaluate the proposed prototype. Collected results highlight ways in which Google interfaces could be modified in order to improve usability for the blind. In our demo we will show how interaction with the modified Google UIs is simplified and how the time for reaching the most important elements (i.e. first query result, next result page, etc.) is shortened in comparison to interaction with the original Google UIs. The demo uses the JAWS screen reader for announcing the UI contents.
\end{abstract}

\section{Categories and Subject Descriptors}

H.5.2 [Information Interfaces and Presentation]: User Interfaces - User-centred design

\section{General Terms \\ Design, Experimentation, Human Factors.}

\section{Keywords}

Search engine, user interface design, accessibility, usability, blind.

\section{INTRODUCTION}

Search engines are particularly difficult for a blind person to use, since difficulties with Web navigation add to the complexity of the search engine interface and functions [1]. Specifically, for people using a screen reader actions take longer and tasks are more difficult since additional actions are required. Craven et al. [2] performed experiments on a sample of blind and visuallyimpaired users who carried out four information-seeking tasks,

Copyright is held by the author/owner(s). ASSETS'06, October 22-25, 2006, Portland, Oregon, USA. ACM 1-59593-290-9/06/0010. including the use of search engines. Visually-impaired users searching the Web for a specific piece of information took on average 2.5 times longer than sighted users. The gap between blind and sighted users' efficiency when performing online search tasks is further explored in [5], where authors discovered that blind participants took twice as long as sighted users to explore search results and three times as long to explore the corresponding web pages. Goble et al. [3] introduced the model of real-world travel to classify the importance of website elements for achieving maximum usability and accessibility, but they do not indicate design guidelines for developers. Recently, Google Labs improved search results based on users' specialized interests. An application of this technology, called Accessible Search, is now available to identify and prioritize search results so they can be more easily used by blind and visually impaired users: regular Google search retrieves a set of documents that is most relevant to user's tasks while Accessible Search helps one find the most accessible pages in that result set. Google Accessible Search analyzes the HTML markup found on a web page and favors accessible pages [4]. In a different way, our prototype acts on the user interfaces for simplifying Google interaction for the blind. Therefore, our approach considers accessibility of the Google UIs rather than accessibility of result pages.

\section{DESIGN AND IMPLEMENTATION OF THE MODIFIED GOOGLE UIS}

Our study shows that it is possible to have a great UI "look \& feel" while assuring satisfaction and efficiency of use for the blind. We chose Google since it is a simple and effective search engine well-known by everyone. We took great care to ensure that the visual appearance of the modified interfaces was identical to the original version. As in a UCD approach, we considered users' problems and needs from the very outset of our study. The main problem is that a blind person does not perceive the overall structure of the interface. To reduce this inconvenience we restructured the HTML code by defining logical sections of the interface. Specifically, we grouped and structured by heading levels sets of homogenous text and elements in order to give the user the overall perception of the interface, and let him/her jump rapidly from one section to another. The home page was divided into four sections, "search box and button", "Advanced Search and Preferences", "Navigation bar" and "Google info", while the most complex result page was subdivided into seven parts, "Results", "Sponsored links", "Result Pages", "Search box and options", "Advanced search and preference links", "Navigation bar" and "Google links" (both pages with sections ordered as 
listed above). Figure 1 shows the JAWS screen reader's interpretation of the original and modified result pages. Italics refer to words/sentences inserted by the screen reader, informing the user about interface elements (link, button, edit field, heading level and so on). New portions, added when we re-engineered the interface, are highlighted in bold. Note how the different order of the sections in the code has changed the screen reader's sequential reading. In the modified UIs the results are the first elements announced. Each result is numbered and separated by a blank line - not visible but captured only by the screen reader -- from the following; whereas in the original UIs there is no clear separation between results, which can be confusing. We also added shortcuts (access keys) and different visiting order (tab keys) to make navigation faster; in addition, short tones have been introduced to help direct attention to the main events such as "focus on the search box", "search successful” and "no result".

\begin{tabular}{|c|c|}
\hline Original Google interface & Modified Google interface \\
\hline Francesco Renga Concerts & $\begin{array}{l}\text { Results for Francesco Renga Concerts } \\
\text { Web }\end{array}$ \\
\hline Link Go to Google Home & Heading level 1 Results: \\
\hline Web & Results 1 - 10 of about 2150 for... \\
\hline Link Images & \\
\hline $\begin{array}{l}\text { Link Groups } \\
\ldots\end{array}$ & $\begin{array}{l}1 \text { Link Northern Italian culture - [...] } \\
\text { http://www.bed-breakfast- }\end{array}$ \\
\hline Edit Francesco Renga Concerts & italy.com/culture/outdoors.htm - 11k - \\
\hline Search Button & Link Cached - \\
\hline $\begin{array}{l}\text { Link Advanced Search } \\
\text { Link Preferences }\end{array}$ & Link Similar pages \\
\hline Search: & 2 Link Live 8 - LIVE on XM [...] \\
\hline Radio button checked the web & $\ldots$ \\
\hline$\ldots$ & Heading level 3 Sponsored Links \\
\hline Web & $\begin{array}{l}\text { Link Francesco Renga CDs [...] } \\
\text { www.cdconnection.com }\end{array}$ \\
\hline Results 1 - 10 of about 2150 for... & Heading level 1 Result Page: \\
\hline Table with 4 columns and 6 rows & 1 \\
\hline Sponsored Links & Link 2 \\
\hline Link Francesco Renga CDs [...] & $\ldots$ \\
\hline www.cdconnection.com & Link Next alt++ \\
\hline table end & $\begin{array}{l}\text { Edit Francesco Renga Concerts alt }+c \\
\text { Search Button }\end{array}$ \\
\hline Link Northern Italian culture $[\ldots]$ & \\
\hline www.bed-breakfast- & Search: \\
\hline $\begin{array}{l}\text { italy.com/culture/outdoors.htm - 11k - } \\
\text { Link Cached - }\end{array}$ & $\begin{array}{l}\text { Radio button checked the Web alt }+w \\
\text {... }\end{array}$ \\
\hline Link Similar pages & Heading level 2 Advanced search: \\
\hline Link Live 8 - LIVE on XM [...] & Link Advanced search $\boldsymbol{a l t}+\boldsymbol{a}$ \\
\hline$\ldots$ & $\begin{array}{l}\text { Heading level } 2 \text { Navigation bar: } \\
\text { Link Navigation help alt }+h\end{array}$ \\
\hline Result Page: & Link Google Homepage $\boldsymbol{a l t}+\boldsymbol{g}$ \\
\hline & Web \\
\hline Link 2 & Link Images \\
\hline$\ldots$ & $\ldots$ \\
\hline Link Next & \\
\hline Edit Francesco Renga Concerts & \\
\hline Search Button ... & \\
\hline
\end{tabular}

Figure 1 - Google UIs interpreted by the screen Reader.

Programmatic access to the search results by Google is provided by the "Google APIs" (http://www.google.com/apis/). When using this APIs, messages are exchanged between the application and Google's server using SOAP (Simple Object Access Protocol), an XML-based messaging that uses HTTP for communication. SOAP messages are short XML messages describing invocations of remote methods. In the case of the Google APIs, there is a method for querying, and a special format for response return.
The XML response is not suitable to be shown directly to endusers, and must be modified via XSLT. Using XSLT, we wrote a "stylesheet" describing how to format each of the elements on the response (title, URL, and text snippet) to be displayed according to the guidelines we proposed [1]. We actually defined two templates: one for the original HTML UIs of Google and the other for the new UIs conform to our design criteria. The total delays from both the SOAP request and the XSLT transformation were not noticeable; the Google API request is sometimes faster than the normal Google Web site, since the size of the transmitted material is smaller. For the transformation we used the Sablotron engine (http://www.gingerall.com/), a fast XML parser and XSLT transformation engine written in $\mathrm{C}$ language. In this way the parsing speed was always less than 0.1 seconds, even when displaying a complex page.

\section{USER FEEDBACK}

To validate our prototype we conducted user testing with 12 totally blind users. Testing itself consisted of 10 tasks including a preliminary exploration of the original and modified Google interfaces, as well as performing free and specific queries and exploring results in both the interfaces. Data collected revealed that all users appreciated the simplified interaction. Specifically, all participants declared that the modified home page interface simplified the search set-up compared to the original one, and 11 of 12 thought the result interface was clearer and easier to use. Concerning the speed of completing the assigned search tasks, 11 of 12 participants acknowledged they felt that the simplified interaction and the greater clarity in result exploration reduced the time needed to carry out the search. The most skilled user stated that the time it took to reach desired results was reduced by 20 $30 \%$ compared to time required for the original Google interface. Regarding evaluation of specific features, participants judged sounds, shortcuts, and different visiting order assigned to links (i.e. by tab index) to be important, as well as hidden labels and numbering of results which assured greater clarity and aided orientation when exploring results.

\section{REFERENCES}

[1] Andronico P., Buzzi M., Castillo, C., Leporini B. Improving Search Engine Interfaces for Blind Users: a Case Study. UAIS Journal, Vol. 5, n. 1 pp. 23-40 (2005), Springer.

[2] Craven, J., Brophy, P. Non-visual access to the digital library: the use of digital library interfaces by blind and visually impaired people. Tech report, Manchester: CERLIM, 2003. http://www.cerlim.ac.uk/pubs/index.php

[3] Goble C., Harper S., Stevens R. The Travails of Visually impaired Web Travellers. In Proceedings of Hypertext '00 (San Antonio, June 2000), ACM Press, 1-10.

[4] Google. Accessible Search FAQ, http://labs.google.com/accessible/faq.html

[5] Ivory, M. Y., Yu, S. Gronemyer, K. Search result exploration: a preliminary study of blind and sighted users' decision making and performance. Extended abstracts of CHI '04 (Vienna, April 2004), ACM Press, 1453-1456. 
